05,13

\title{
Затухание спиновой прецессии в гетероструктурах манганит/нормальный металл
}

\author{
(C) Т.А. Шайхулов, Г.А. Овсянников
}

Институт радиоэлектроники им. В.А. Котельникова РАН, Москва, Россия

E-mail: shcaihulov@hitech.cplire.ru

\begin{abstract}
Изучена температурная зависимость затухания магнитной спиновой прецессии в двухслойных структурах с верхним слоем Pt на основе эпитаксиальной пленки манганита $\mathrm{La}_{0.7} \mathrm{Sr}_{0.3} \mathrm{MnO}_{3}$ (LSMO) путем измерения ширины линии спектра ферромагнитного резонанса (ФМР). Ферромагнитный резонанс в тонких ферромагнитных пленках манганита используется для создания спинового тока на границе раздела между металлическим и ферромагнитным слоями. Обсуждается значительное увеличение ширины линии в спектре ФМР в двухслойных структурах за счет генерации спинового тока, неоднородности ферромагнитного слоя, двухмагнонного рассеяния и вихревого тока.
\end{abstract}

Работа выполнена при частичной поддержке гранта РФФИ 18-37-00170.

DOI: $10.21883 /$ FTT.2018.11.46662.22NN

\section{1. Введение}

Работа устройств спинтроники основана на переносе спина в магнитных гетероструктурах, связанных с переносом спинового момента. Магнитная гетероструктура, как правило, состоит из магнитного и немагнитного слоев. Редкоземельные манганитовые перовскиты со структурой $\operatorname{Re}_{1-x} A_{x} \mathrm{MnO}_{3}(R e-$ редкоземельные материалы типа $\mathrm{La}$ или $\mathrm{Nd})$, a $A$ - щелочноземельные металлы, такие как $\mathrm{Sr}, \mathrm{Ca}, \mathrm{Ba})$ проявляют широкий спектр необычных электрических и магнитных свойств, включая высокую (до 100\%) магнитную поляризацию, эффект колоссального магнитосопротивления и др. (см. обзор [1]). Параметры эпитаксиальных пленок этих материалов очень сильно отличаются от свойств монокристаллов. Значительное влияние на магнитные и электрические свойства пленок обусловлено деформацией пленок, вызванных несоответствием с подложкой, на которой осаждается манганитная пленка [1-3]. Эффекты разделения фаз и наличие немагнитного слоя на границе раздела подложки/пленки могут проявляться в очень тонких пленках (менее $10 \mathrm{~nm}$ ). [4] Манганиты лантана-стронция $\mathrm{La}_{0.7} \mathrm{Sr}_{0.3} \mathrm{MnO}_{3}$ (LSMO) имеют высокую спиновую поляризацию (до 100\%) и могут быть использованы в магнитных туннельных переходах [5] и спиновых клапанах [6-8]. Манганитные пленки, для которых температура Кюри $T_{C}$ близка к комнатной температуре, особенно привлекательна для практического применения. Хотя был проведен ряд исследований по возбуждению спинового тока ферромагнитным резонансом в структурах LSMO/N ( $N$ - нормальный металл, обычно платина) $[9,10]$, нет данных о температурных зависимостях ширины линии ферромагнитного резонанса (ФМР) от спинового тока в ферромагнетиках и влиянии других источников ширины линии, таких как неоднородность ферромагнитного слоя, двухмагнонное рассеяние и вихревые токи.

\section{2. Модель затухания спиновой прецессии}

Затухание Гильберта $\alpha$ является мерой релаксации спиновой прецессии в однородных ферромагнетиках за счет спин-орбитального взаимодействия [12]. Ширина линии ФМР, индуцированная затуханием Гильберта при измерении ФМР, пропорциональна частоте ФМР $\omega$ $\Delta H_{G}=\alpha \omega / \gamma\left(\gamma=g \mu_{\mathrm{B}} / h-\right.$ гиромагнитное отношение $)$ и описывает ситуацию для однородного случая. В ферромагнитной структуре из ферромагнетика и нормального металла линия ФМР дополнительно уширяется за счет генерации спинового тока, неоднородности намагниченности ферромагнетика, взаимодействием с другим материалом, двухмагнонным рассеянием и возникновением вихревого тока в ферромагнетике. Экспериментально измеренная ширина линии FMR $\Delta H_{P P}$ может быть представлена в виде суммы

$$
\Delta H_{P P}=\Delta H_{G}+\Delta H_{I}+\Delta H_{2 M}+\Delta H_{E},
$$

где $\Delta H_{I}, \Delta H_{2 M}, \Delta H_{E}$ - ширина линий для затухания, вызванного неоднородным состоянием ферромагнетика, двухмагнонным рассеянием и затуханием, вызванным вихревым током соответственно $[13,14]$. Изменение магнитных свойств материалов, таких как его анизотропия или намагниченность влияет на увеличение ширины линии $\Delta H_{I}$, которая не зависит от частоты $[13,14]$. Магнитное поле переменного тока, вызванное ФМР, индуцирует вихревые токи в тонкой пленке. Эти токи производят дополнительное изменение амплитуды магнитных полей переменного тока гетероструктуры. Влияние вихревых токов на ферромагнитный резонанс в проводимой ферромагнитной системе может привести к уширению ширины линии ФМР и изменению формы спектра ФМР в неоднородной области микроволновых полей [15-17]. Механизм даухмагнонного рассеяния приводит к связи 
режима равномерной прецессии с $k=0$, возбужденной ФМР, с вырожденными конечно спин-волновыми модами $[17,18]$. Увеличение параметра затухания Гильберта в гетероструктуре ферромагнетик/нормальный металл, вызванное прецессией намагниченности в ферромагнетике, вызывает протекание спинового тока через границу в нормальный металл [19]. Теория [19] предсказывает протекание спинового тока от ферромагнитного до немагнитного слоя перпендикулярно границы раздела

$$
j_{s}^{0} \mathbf{s}=\frac{\hbar}{8 \pi} \operatorname{Re}(2 g \uparrow \downarrow)\left[\mathbf{m} \times \frac{\partial m}{\partial t}\right],
$$

где $m=M / M_{S}$ - единичный вектор намагничивания ферромагнитного слоя, $\operatorname{Re}(2 g \uparrow \downarrow)$ - спиновая проводимость границы раздела, которая аддитивно добавляется к компонентам затухания Гильберта. Затухающий параметр Гильберта можно записать в виде: $\alpha=\alpha_{0}+\alpha^{\prime}$, где $\alpha_{0}-$ собственный вклад и $\alpha^{\prime}$ является дополнительным затуханием, обусловленным спиновой накачкой [19-23].

\section{3. Образцы и экспериментальная техника}

Эпитаксиальные пленки $\mathrm{La}_{0.7} \mathrm{Sr}_{0.3} \mathrm{MnO}_{3}$ (LSMO) осаждались магнетронным распылением на монокристаллические подложки (110) $\mathrm{NdGaO}_{3}$ (NGO) при $T=820^{\circ} \mathrm{C}$
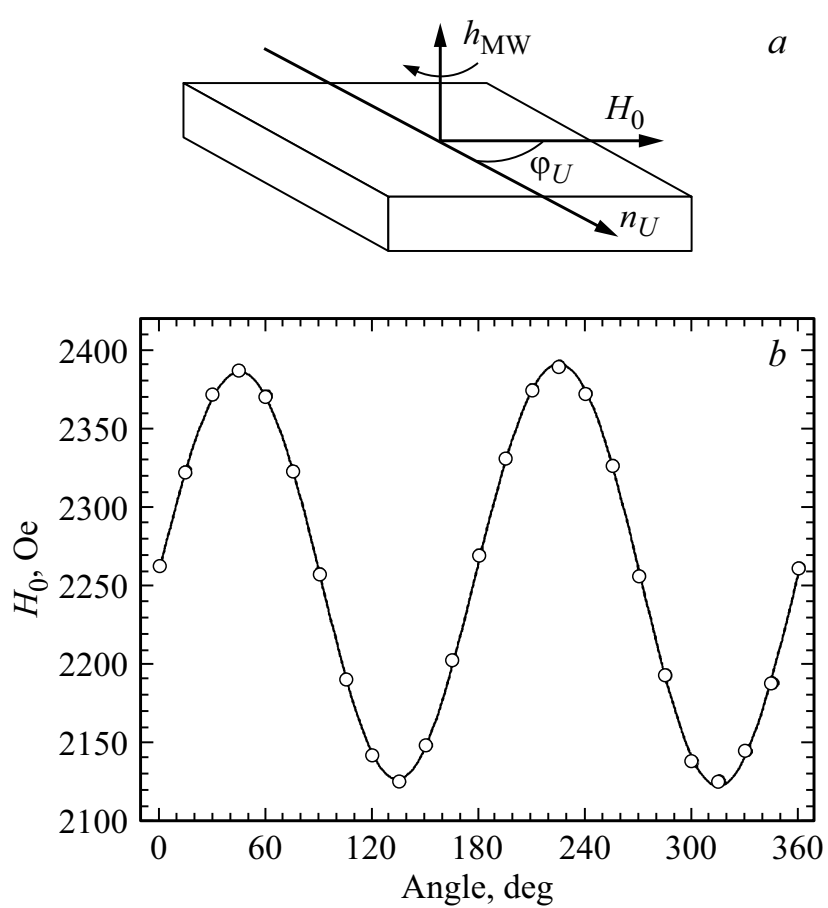

Рис. 1. $a-$ ориентация образца относительно направлений постоянного и СВЧ-полей. $b-$ угловая зависимость ширины линии резонансного поля для пленки $h$-LSMO при $T=300 \mathrm{~K}$. Подгонка экспериментальных данных дает следующие магнитные параметры пленки LSMO: намагниченность $M_{0}=300$ Oе, $H_{U}=190 \mathrm{Oe}, H_{C}=10 \mathrm{Oe}$. и давлении кислорода 0.15-1 mbar. 10-20 nm Pt или $\mathrm{Au}$ напылялись ex situ сразу после охлаждения пленки. Контактные площадки были получены путем распыления Pt через металлическую маску. Сопротивление пленок изучалось четырехточечным методом, что исключает влияние контактного сопротивления.

Магнитные характеристики измеряли методом магнитного резонанса с использованием спектрометра фирмы Bruker (частота $9.51 \mathrm{GHz}$ ). Исследованные образцы располагались в микроволновой полости спектрометра таким образом, чтобы плоскость образца всегда была параллельна направлению постоянного внешнего магнитного поля и магнитной составляющей СВЧ-поля (параллельная ориентация). Такое расположение образцов устранило изменение спектров магнитного резонанса изза размагничивающего фактора формы образца. Вращение образцов проводилось вокруг оси, перпендикулярной плоскости образцов (см. рис. 1,a). Были исследованы пленки сразу после осаждения до комнатной температуры ( $d$-LSMO) и отожженные после роста при $T=820^{\circ} \mathrm{C}$ в течение одного часа ( $h$-LSMO).

\section{4. Экспериментальные результаты и обсуждение}

\section{1. Магнитные параметры пленок LSMO}

Параметры магнитной анизотропии определялись из обработки угловых зависимостей резонансных полей спектров ФМР (см. рис. $1, b)$. Используется решение уравнения Ландау-Лифшица для эволюции намагниченности $M$ во внешнем постоянном магнитном поле $H$ под действием магнитной составляющей радиочастотного поля, которое дает аналитическое уравнение для резонансного поля $H_{0}$ и частоты $\omega$ [24].

На рис. $1, b$ показана угловая зависимость резонансного значения магнитного поля $H_{0}$ для пленки $h$-LSMO, измеренная при комнатной температуре, при повороте вокруг нормали к плоскости пленки на угол $\varphi$, который измерялся от одной из граней подложки (обозначенной $n_{u}$ на рис. $1, a)$. Поскольку подложка с пленкой имела площадь $5 \times 5 \mathrm{~mm}$, эффект анизотропии формы образца минимален, а весь сдвиг резонансного поля обусловлен магнитной анизотропией в плоскости пленки LSMO. Экспериментально измеренная угловая зависимость хорошо описывалась резонансным соотношением с учетом одноосной, вызванной влиянием анизотропии подложки, и двухосной (кубической) анизотропиями [24]. В результате были определены намагниченность $M_{0}$, а также $K_{u}$ и $K_{c}$ - константы одноосной и двухосной анизотропии соответственно, поля которых определяются следующим образом: $H_{u, c}=2 K_{U, C} / M_{0}$.

Температурные зависимости магнитных параметров $h$-LSMO пленки показаны на рис. 2. Намагниченность пленки увеличивается до $2.2 \mu_{\mathrm{B}} / M n$ при $T \leq 200 \mathrm{~K}$. Поля магнитной анизотропии возрастают с понижение 


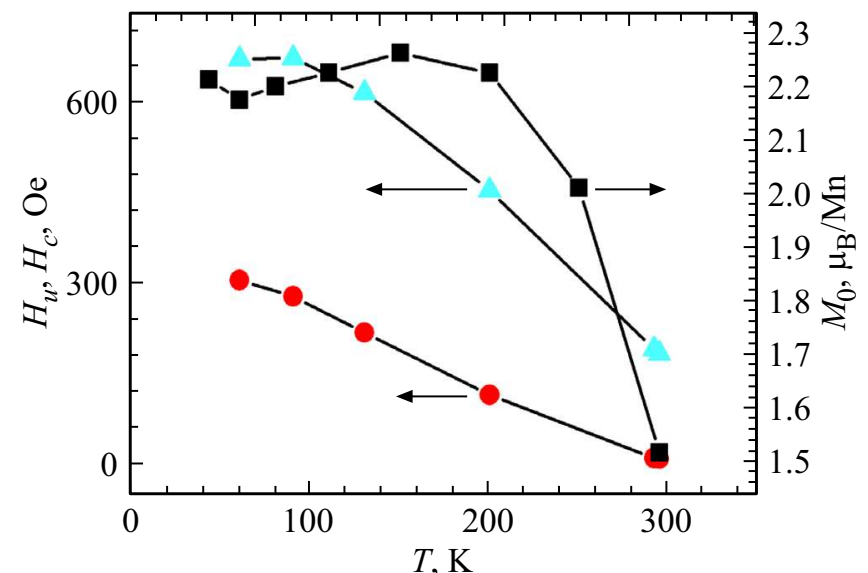

Pис. 2. Температурная зависимость намагниченности $M_{S}$ (черные квадраты), двухосная $H_{u}$ (светлые треугольники) и кубическая $H_{c}$ (темные кружки) анизотропии магнитного поля соответственно для $h$-LSMO-пленки.

температруры $T<50 \mathrm{~K}$. Видно, что одноосная магнитная анизотропия, индуцированная орторомбичностью подложки (110) $\mathrm{NdGaO}_{3}$, доминирует над внутренней двухосной кубической анизотропией $\left(H_{C}\right)$.

\section{2. Однородное затухание}

Ширина линии ферромагнитного резонанса (ФМР) $\Delta H$, измеренная при сканировании внешнего магнитного поля $H$, определяется как разность положений по полю между экстремумами $H_{p+}, H_{p-}$ первой производной $d P / d H$ сигнала поглощения СВЧ-поля (см. рис. 3). При этом значении резонансное поле $H_{0}$, определяемое как точка перехода сигнала $d P / d H$ через нуль, всегда находится в диапазоне $H_{p+}<H_{0}<H_{p-}$. Заметим, что определение ширины линии путем аппроксимации спек-

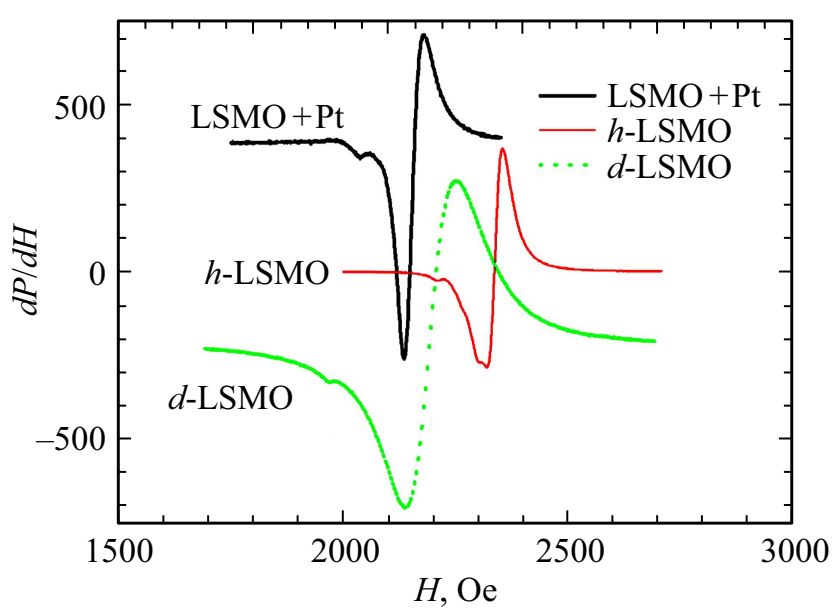

Рис. 3. Спектры ФМР для пленок $d$-LSMO и $h$-LSMO и гетероструктур Pt $/ h$-LSMO. Спектры для $d$-LSMO и Pt $/ h$-LSMO смещены по оси $d P / d H$. тра ФМР несколькими линиями Лоренца дает примерно $10 \%$ поправку в величине $\Delta H_{P P}$.

Для пленок $h$-LSMO толщиной $40 \mathrm{~nm}$ находим затухание спиновой прецессии при комнатной температуре из ширины линии $\Delta H_{P P}=28 \mathrm{Oe}, \alpha_{0}=\Delta H_{P P} \gamma / \omega=8 \cdot 10^{-3}$. При толщине $\mathrm{Pt}$ равной $10 \mathrm{~nm}$, нанесенной на пленку $h$-LSMO $\alpha$ увеличивается на 10\%. Увеличение затухания при напылении Pt на пленку LSMO $\alpha=\alpha_{0}+\alpha^{\prime}$ может возникнуть из-за протекания спинового тока $[25,26]$ через границу Pt/LSMO. Используя [25,26] можно вычислить спиновую проводимость в гетероструктуре $\mathrm{Pt} / \mathrm{LSMO}$

$$
g_{\mathrm{eff}}^{\uparrow \downarrow}=\frac{4 \pi M_{s} t_{\mathrm{LSMO}}}{g \mu_{\mathrm{B}}} \alpha^{\prime},
$$

$\gamma=17.605 \cdot 10^{6}$ - гиромагнитное отношение для электрона, $\omega=2 \pi \cdot 9.51 \cdot 10^{9} \mathrm{~s}^{-1}-$ микроволновая угловая частота, $M_{s}=300 \mathrm{Oe}-$ намагниченность пленки $\mathrm{LSMO}, t_{\mathrm{LSMO}}=40 \mathrm{~nm}$ - толщина пленки LSMO слоя, $\mu_{\mathrm{B}}=9.274 \cdot 10^{-21} \mathrm{erg} / \mathrm{G}$ - магнетон Бора, $g=2$ фактор Ланде. При комнатной температуре мы получаем увеличение ширины линии ФМР после напыления $\mathrm{Pt} \Delta H_{\mathrm{Pt} / \mathrm{LSMO}}-\Delta H_{\mathrm{LSMO}}=4 \mathrm{Oе}$ и, следовательно, $g_{\text {eff }}=0.4 \cdot 10^{19} \mathrm{~m}^{-2}$. Эта величина спиновой проводимости границы несколько превышает $g_{\text {eff }} \sim 10^{18} \mathrm{~m}^{-2}$, полученную из измерений на наших структурах Pt/LSMO спинового тока с помощью обратного спинового эффекта Холла [10]. Для сравнения для границ $\mathrm{Py} / \mathrm{Pt}$ было получено $g_{\text {eff }}=2.1 \cdot 10^{19} \mathrm{~m}^{-2}$ [27], а для $\mathrm{YIG} / \mathrm{Pt}$ $g_{\text {eff }}=4.8 \cdot 10^{20} \mathrm{~m}^{-2}[28]$.

При оценке спиновой проводимости, используя (3), не учитываются другие механизмы затухания спиновой прецессии. Эффективная одномерная спиновая проводимость нормального металлического слоя, соединенная последовательно со спиновой проводимостью границы

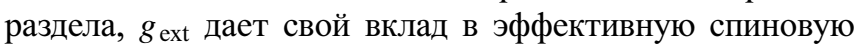
проводимость [23,29]:

$$
g_{\uparrow \downarrow}^{\text {eff }}=\left(1 / g_{\uparrow \downarrow}+1 / g_{\text {ext }}\right) .
$$

Функциональная форма $g_{\text {ext }}$ получается путем решения уравнения спиновой диффузии с соответствующими граничными условиями. В случае структуры ферромагнетик/нормальный металл получено следующее выражение для спиновой проводимости [26]:

$$
g_{\text {ext }}=\tanh \left(d_{\mathrm{Pt}} / \lambda_{d}\right) /\left(2 \lambda_{d} \rho_{\mathrm{Pt}}\right)
$$

где $\rho_{\mathrm{Pt}}, d_{\mathrm{Pt}}$ и $\lambda_{d}-$ удельное сопротивление, толщина и длина диффузии для пленки $\mathrm{Pt}$ соответственно. Для $d_{\mathrm{Pt}}=10 \mathrm{~nm}$ больше, чем $\lambda_{d}=3 \mathrm{~nm}$ [26] $\left.\tanh \left(d_{\mathrm{Pt}} / \lambda\right)_{d}\right) \approx 1$, вклад в ширину линии от спиновой проводимости в пленке Pt равен при комнатной температуре

$$
\Delta H_{\mathrm{ext}}=(\omega / \gamma) g \mu_{\mathrm{B}} h /\left(2 \pi 4 e^{2} M_{s} d_{\mathrm{F}} \rho_{\mathrm{Pt}} \lambda_{d}\right) \approx 6 \mathrm{Oe},
$$

где $g=2, \quad M_{s}=300 \mathrm{Oe}, \quad d_{\mathrm{F}}=4 \cdot 10^{-6} \mathrm{~cm}, \quad \rho_{\mathrm{Pt}}=$ $=3 \cdot 10^{-5} \Omega \cdot \mathrm{cm}, \quad \lambda_{d}=3 \cdot 10^{-7} \mathrm{~cm}, \quad h / e^{2}=2.6 \cdot 10^{4} \Omega$. 
Уширение линии ФМР за счет эффективной спиновой проводимости нормального металлического слоя оказывается равным вкладу от спинового тока. Большое значение этого уширения возможно вызвано ошибкой в определении диффузионной длины $\lambda_{d}$.

\section{3. Вихревой ток}

Температурные зависимости ширины линии для пленки манганита $h$-LSMO и для гетероструктуры Pt $/ h$-LSMO представлены на рис. 4. С понижением температуры $\Delta H_{p p}$ заметно увеличивается. Увеличение $M_{s}$ с понижением $T$ может стать причиной увеличения ширины линии (см. выражение (3)). Но ниже $T=200 \mathrm{~K} M_{s}$ насыщается (см. рис. 2), а ширина линии ФМР продолжает расти.

Увеличение проводимости LSMO-пленки с понижением $T$ может увеличить ширину линии за счет образования вихревого тока в пленках. Вихревой ток связан с потерей энергии из-за связи с электронами проводимости без привлечения тепловых магнонов. В случаях, когда глубина скин-слоя является большой по отношению к размеру структуры, потери на вихревые токи зависят от размера образца, проводимости и частоты $[15,16,30]$.

Влияние вихревых токов в тонких пленках LSMO и Pt определяется из расчетов уширения линии ФМР тонкого диска с радиусом $r$ и толщиной $d[30]$ :

$$
\Delta H_{E}=k^{2} d^{2} \varepsilon^{\prime \prime} 4 \pi M_{s} / 10\left(1-(3 / 25) k^{2} r\right),
$$

где $\varepsilon^{\prime \prime}=4 \pi \sigma / \omega, k=2 \pi / \lambda-$ постоянная свободного пространства, $M_{s}=300 \mathrm{Oe}$ - магнитная пленочная намагниченность, $\sigma$ - проводимость ферромагнетика. При $\lambda=3.16 \mathrm{~cm}$ и $r \approx 0.1 \mathrm{~cm},(3 / 25) k^{2} r \ll 1$ выражение для влияния вихревого тока на ширину линии выглядит

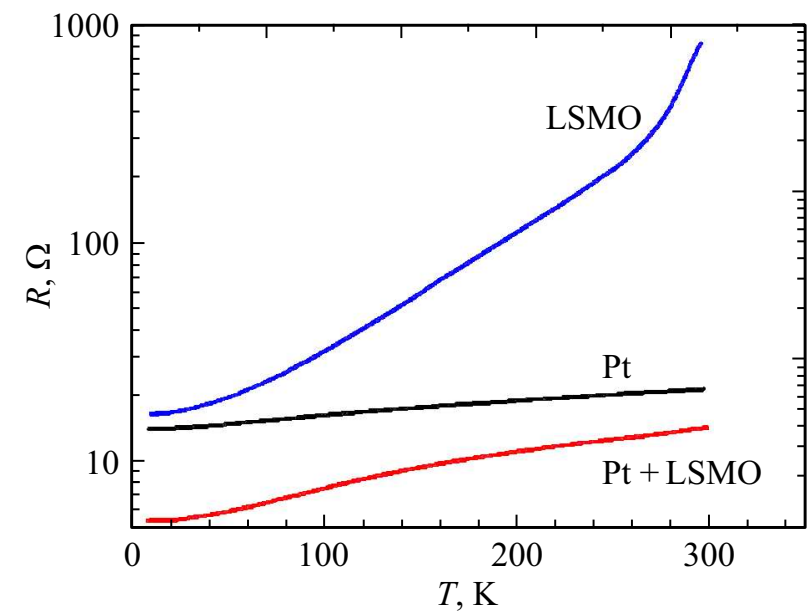

Рис. 4. Температурная зависимость сопротивления пленок Pt и $h$-LSMO, а также гетероструктуры Pt/h-LSMO. Удельные сопротивления пленок $\mathrm{Pt}$ в автономном случае (напыления на подложку) и для гетероструктуры различаются из-за разных толщин и типов роста.

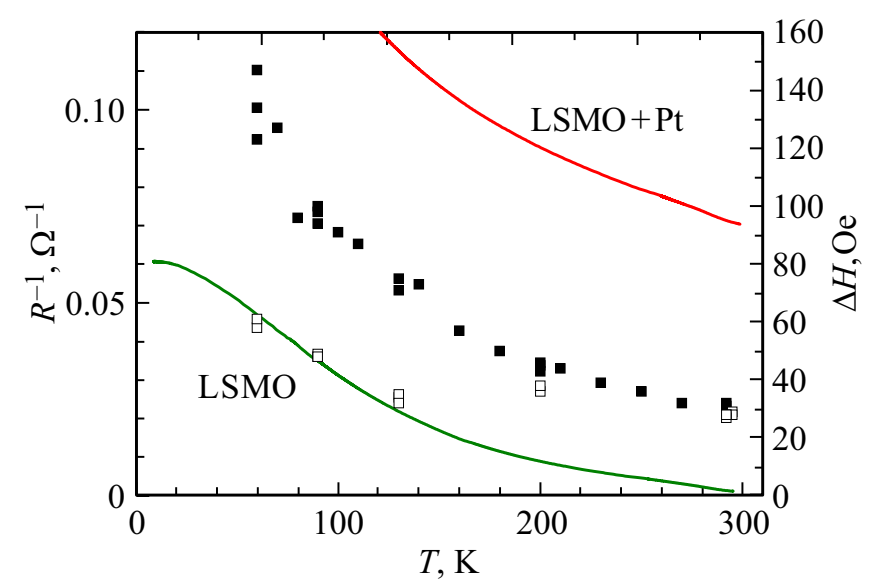

Рис. 5. Температурная зависимость ширины линии ФМР для пленки $h$-LSMO (светлые прямоугольники) и гетероструктуры $\mathrm{Pt} / h$-LSMO (темные прямоугольники) Сплошными линиями показаны зависимости обратного сопротивления структур.

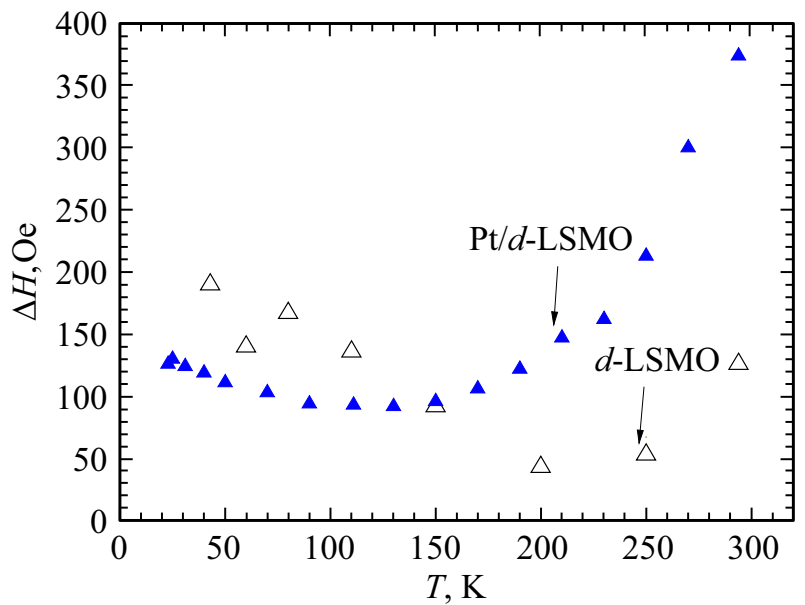

Рис. 6. Температурная зависимость ширины линии ФМР для гетероструктуры Pt/d-LSMO (черный треугольник) и пленки $d$-LSMO (светлый треугольник) для двух углов направлений магнитного поля.

следующим образом

$$
\Delta H_{E}=(4 \pi)^{2} d^{2} M_{s} \omega \sigma /\left(10 c^{2}\right),
$$

где $c$ - скорость света. Для пленки LSMO с параметрами $d=40 \mathrm{~nm}, \omega=2 \pi 9.51 \cdot 10^{9} \mathrm{~s}^{-1}, \sigma=300(\Omega \cdot \mathrm{cm})^{-1}$, вклад от вихревого тока в ширину линии FMR мал и составляет $\Delta H_{E}=2$ Ое при комнатной температуре. С понижением температуры проводимость LSMO-пленки растет и пропорционально увеличивается ширина линии ФМР (см. рис. 5). Если при комнатной температуре вклад вихревого тока в ширину линии ФМР LSMO-пленки $\Delta H_{p p}$ мал, то с уменьшением температуры $T<150 \mathrm{~K}$ он увеличивается пропорционально изменению проводимости ферромагнетика, как следует из (8). При азотной температуре ширина линии ФМР в два раза больше, чем $\Delta H_{p p}$ при комнатной температуре. 
При осаждении пленки Pt поверх пленки LSMO общая проводимость структуры увеличивается (см. рис. 4). Увеличение $\Delta H_{p p}$, которое наблюдается в эксперименте после напыления $\mathrm{Pt}$, вполне можно объяснить генерацией спинового тока в Pt/LSMO гетероструктуре. C понижением температуры удельное сопротивление пленки $\mathrm{Pt}$ уменьшается пропорционально $T$, а сопротивление LSMO-пленки изменяется более чем на один порядок. Вклад всех слоев в удельном сопротивлении гетероструктуры объясняется тем, что пленка LSMO вместе с пленкой Pt выступает в качестве параллельных резисторов [31].

\section{4. Неоднородное затухание}

Неоднородный вклад в уширение линии ФМР связан с магнитным беспорядком в пленке. Изменение магнитных свойств материалов, таких как его анизотропия или намагниченность, приводит к уширению линии, нелинейно зависит от частоты. Вклад в ширину линии $\Delta H_{I}$ зависит от неоднородности образца, которая связана с локальным изменением направления и амплитуды намагниченности. Неоднородное уширение ширины линии $\Delta H_{I}$ можно описать как

$$
\Delta H_{I}=\left|\partial H_{r} / \partial \varphi\right| \Delta \varphi+\left|\partial H_{r} / \partial(M)\right| \Delta(M),
$$

где $\Delta \varphi_{H}$ и $\Delta(M)$ - разброс ориентации кристаллографических осей и амплитуды намагниченности соответственно $[14,32,33]$. Влияние неоднородности наблюдается для пленок $d$-LSMO при $T$ вблизи $T_{C}$ (см. рис. 5). Вблизи температуры Кюри наблюдалось сильное увеличение $\Delta H_{p p}$ для переходных металлов, таких как Ni и $\mathrm{Fe}[14,34]$. Возможными причинами увеличения ширины линии вблизи $T_{C}$ является рассеяние на границах и неоднородностях в пленках [35]. Для отожженых пленок $h$-LSMO нет увеличения ширины линии вблизи $T_{C}$. Поскольку ширина линии LSMO-пленки уменьшилась после нагревания, мы считаем, что пленка $h$-LSMO стала более однородной. Влияние неоднородного уширения линии, вызванного разбросом параметров уменьшилось. В последнем случае пик ширины линии вблизи $T_{C}$ отсутствует. Ниже температуры Кюри наблюдалось минимум $\Delta H_{p p}$ для пленок $d$-LSMO около $0.6 T_{C}$. Качественно увеличение ширины линии при более низкой температуре можно понимать как неоднородное уширение из-за влияния анизотропии магнитной пленки. Известно, что константы анизотропии сильно зависят от температуры и увеличиваются с уменьшением температуры.

Неоднородность пленки может вызвать режим прецессии намагниченности $(k=0)$, возбуждаемый в эксперименте ФМР, для вырождения конечных мод $k \neq 0$ спиновых волн. Этот механизм релаксации однородной моды известен как рассеяние магнонов [32,36]. Двухмагнонный процесс основан на модели, в которой один магнон однородной прецессии аннулируется, а создается еще один магнон с той же энергией и ненулевым волновым вектором, называемым вырожденным магноном. Рассеяние от однородной прецессии к вырожденной моде является важным источником релаксации в магнитных материалах. Это негильбертовский механизм затухания в кристаллических магнитных пленках.

Поведение ширины линии ФМР, описываемое двумагнонным рассеянием, показывает, что процесс нелинейный по $\omega$. Если $f \ll f_{M}$ (ниже характерной частоты $\left.f_{M}=\left(g \mu_{\mathrm{B}} / h\right) M_{S}\right)$, то ширина линии должна линейно меняться с частотой, аналогично предсказанию затухания Гилберта. Однако, если $f \sim f_{M}$, он проявляет существенные отклонения от линейного поведения и насыщается на высоких частотах $f \gg f_{M}[18,32,35]$.

Приведенная ниже угловая зависимость $\Delta H_{p p}$ с учетом кубической анизотропии учитывает двухмагнонное рассеяние $[14,37]$ :

$$
\begin{aligned}
\Delta H_{p p}= & \Delta H_{0}+\alpha \omega / \gamma+\Delta H_{2 m}^{100} \cos ^{2}(2 \varphi) \\
& +\Delta H_{2 m}^{110} \cos ^{2}(2[\varphi-\pi / 4]) .
\end{aligned}
$$

Данные ширины линии могут иметь параметры $\Delta H_{0}, \alpha$ и $\Gamma_{2 m}$. Сравнение данных для LSMO-пленки и гетероструктуры Pt/LSMO показывает, что Pt покрывая пленку LSMO, увеличивет параметр двухмагнонного рассеяния. В нашем эксперименте не наблюдается предсказанной выражением (10) угловой зависимости ширины линии ФМР. Возможно здесь проявляется доминирование одноосной магнитной анизотропией.

\section{5. Заключение}

Исследована температурная зависимость ширины линии ФМР и магнитных параметров пленок LSMO и гетероструктур Pt/LSMO. Наблюдалась немонотонная зависимость от температуры ширины линии спектра ФМР для пленки $d$-LSMO. Ширина линии при высокой температуре сначала уменьшается с понижением температруы, а потом возрастает при $T<150 \mathrm{~K}$. Высокое значение ширины линии при комнатной температуре может быть объяснено неоднородностью магнитной структуры для $d$-LSMO пленки и увеличением ширины линии для гетероструктуры Pt/d-LSMO за счет двухмагнонного механизма затухания прецессии спина. Для отожженных пленок $h$-LSMO наблюдается только увеличение ширины линии при понижении температуры. Наблюдалось аномальное увеличение ширины линии спектра ФМР для эпитаксиальных пленок LSMO после осаждения пленки Pt сверху. Увеличение ширины линии в гетероструктуре Pt/h-LSMO при низких температурах $T<150 \mathrm{~K}$ можно объяснить уменьшением сопротивления пленки и вкладом механизма уширения ширины линии ФМР за счет возбуждения вихревого тока.

Авторы благодарны В.В. Демидову и В.А. Ацаркину за полезное обсуждение полученных результатов и помощь при проведении исследований. 


\section{Список литературы}

[1] A.-M. Haghiri-Cosnet, J.P. Renard. J. Phys D 36, R127 (2003).

[2] Zh. Huang, G.Y. Gao, Zh.Zh. Yin, X.X. Feng, Y.Zh. Chen, X.R. Zhao, J.R. Sun, W.B. Wu. J. Appl. Phys. 105, 113919 (2009).

[3] G.A. Ovsyannikov, A.M. Petrzhik, I.V. Borisenko, A.A. Klimov, Yu.A. Ignatov, V.V. Demidov, S.A. Nikitov. JETP 108, 48 (2009).

[4] N.D. Mathur, G. Burnell, S.P. Isaac, T.J. Jackson, B.S. Teo, J.L. MacManus-Driscoll, L.F. Cohen, J.E. Evetts, M.G. Blamire. Nature 387, 266 (1997).

[4] M. Bowen, M. Bibes, A. Barthélémy, J.P. Contour, A. Anane, Y. Lemaitre, A. Fert. Appl. Phys. Lett. 82, 233 (2003).

[6] Y. Ishii, H. Yamada, H. Sato, H. Akoh, M. Kawasaki, Y. Tokura. Appl. Phys. Lett. 87, 22509 (2005).

[7] Z.H. Xiong, D. Wu, Z.V. Vardeny, J. Shi. Nature 427, 821 (2004).

[8] V. Dediu, M. Murgia, F.C. Matacotta, C. Taliani, S. Barbanera. Solid State Commun 122, 181 (2002).

[9] G.Y. Luo, C.R. Chang, J.G. Lin. J. Appl. Phys. 115-119, 17C508 (2014).

[10] V.A. Atsarkin, B.V. Sorokin, I.V. Borisenko, V.V. Demidov, G.A. Ovsyannikov. J. Phys. D 49, 125003 (2016).

[11] T.L. Gilbert. IEEE Trans. Magn. 40, 3443 (1949).

[12] S.V. Vonsovskii. Ferromagnetic Resonance. Academic, N.Y. (1966).

[13] T.G.A. Verhagen, H.N. Tinkey, H.C. Overweg, M. van Son, M. Huber, J.M. van Ruitenbeek, J. Aarts. J. Phys. Condens. Matter 28, 056004 (2016).

[14] W. Platow, A.N. Anisimov, G.L. Dunifer, M. Farle, K. Baberschke. Phys. Rev. B 58, 5611 (1998).

[15] V. Flovik, F. Macia, A.D. Kent, E. Wahlström. J. Appl. Phys. 117, 143902 (2015).

[16] A.G. Flores, M. Zazo, V. Raposo, J. Iniguez. JAP 93, 8068 (2003).

[17] B. Heinrich, R. Urban, G. Woltersdorf. J. Appl. Phys. 91, 7523 (2002).

[18] J.-M. Beaujour, D. Ravelosona, I. Tudosa, E.E. Fullerton, A.D. Kent. Phys. Rev. B 80, 180415 (2009).

[19] A. Tserkovnyak, Brataas, E.W. Bauer. Phys. Rev. Lett. 88, 117601 (2002).

[20] A. Azevedo, L.H. Vilela-Leao, R.L. Rodriguez-Suarez, A.F. Lacerda Santos, S.M. Rezende. Phys. Rev. B 83, 144402 (2011).

[21] O. Mosendz, V. Vlaminck, J.E. Pearson, F.Y. Fradin, G.E.W. Bauer, S.D. Bader, A. Hoffmann. Phys. Rev. 82, 214403 (2010).

[22] M. Rezende, R.L. Rodriguez-Suarez, M.M. Soares, L.H. Vilela-Le, D. Ley Dominguez, A. Azeved. Appl. Phys. Lett. 102, 012402 (2013).

[23] S. Emori, U.S. Alaan, M.T. Gray, V. Sluka, Y. Chen, A.D. Kent, Y. Suzuki. Phys. Rev. B 94, 224423 (2016).

[24] V.V. Demidov, G.A. Ovsyannikov, A.M. Petrzhik, I.V. Borisenko, A.V. Shadrin, R. Gunnarsson. J. Appl. Phys. 113, 163909 (2013).

[25] G.Y. Luo, M. Belmeguenai, Y. Roussigné, C.R. Chang, J.G. Lin, S.M. Cherif. AIP Adv. 5, 097148 (2015).

[26] J.-C. Rojas-Sanchez, N. Reyren, P. Laczkowski, W. Savero, J.-P. Attane, C. Deranlot, M. Jamet, J.-M. George, L. Vila, H. Jaffrés. Phys. Rev. Lett. 112, 106602 (2014).
[27] O. Mosendz, V. Vlaminck, J.E. Pearson, F.Y. Fradin, G.E.W. Bauer, S.D. Bader, A. Hoffmann. Phys. Rev. 82, 214403 (2010).

[28] M. Rezende, R.L. Rodriguez-Suarez, M.M. Soares, L.H. Vilela-Le, D. Ley Dominguez, A. Azeved. Appl. Phys. Lett. 102, 012402 (2013).

[29] C.T. Boone, H.T. Nembach, J.M. Shaw, T.J. Silva. J. Appl. Phys. 113, 153906 (2013).

[30] M. Marysko. Phys. Status Solidi A 28, 159 (1975).

[31] C.T. Boone, J.M. Shaw, H.T. Nembach, T.J. Silva. J. Appl. Phys. 117, 223910 (2015).

[32] C. Luo, Z. Feng, Y. Fu, W. Zhang, P.K.J. Wong, Z.X. Kou, Y. Zhai, H.F. Ding, M. Farle, J. Du, H.R. Zhai. Phys. Rev. B 89, 184412 (2014).

[33] C. Chappert, K.L. Dang, P. Beauvillain, H. Hurdequint, D. Renard. Phys. Rev. B 34, 3192 (1986).

[34] Yi Li, K. Baberschke. Phys. Rev. Lett. 68, 1208 (1992).

[35] Å. Monsen, J. Boschker, F. Macià, J. Wells, P. Nordblad, A.D. Kent, R. Mathieu, T. Tybell, E. Wahlström. J. Magn. Magn. Mater 369, 197 (2014).

[26] R. Arias, D.L. Mills. Phys. Rev. B 60, 7395 (1999).

[37] G. Woltersdorf, B. Heinrich. Phys. Rev. B 69, 184417 (2004).

Редактор К.В. Емиев 